\title{
HIV I-2 Ag/Ab selective and combined detection by a new rapid point-of-care test
}

\author{
Marta Monari, Serenella Valaperta, Eleonora Baldassarre, Roberto Assandri, Alessandro Montanelli \\ Clinical Investigations Laboratory, IRCCS Istituto Clinico Humanitas, Rozzano (MI)
}

Key words: HIV, exposure, POCT

Point-of-care test per la ricerca di HIV I-2 Ag/Ab: rapidità e accuratezza

\section{SUMMARY}

In order to reduce the window phase between time of human immunodeficiency virus (HIV) exposure and source's results of laboratory investigations, we evaluated the analytical performance of a new point of care test for simultaneous detection of HIV Abs and HIV Ag. We tested 48 serum samples by HIV Combo Ag/Ab Abbott Diagnostic ${ }^{\circledast}$, USA; by Western Blot Matrix HIV I/2 Abbott ${ }^{\circledast}$, USA and by the new POCT test HIV I/2 Ag/Ab Combo Inverness Medical ${ }^{\circledR}$, UK. Good concordance of results was recordered with analytical performance (sensitivity and specificity) and diagnostic accuracy of $100 \%$. Thus this new test is ideal for rapid laboratory investigation when the time for appropriate prophylactic therapy is very important, such as found in exposure in the workplace.

\section{BACKGROUND}

Despite ongoing prevention and educational efforts, an estimated 4.000 new HIV infections occur annually in Italy (7). The typical evolution of viral and host markers of HIV infection are presented in Figure I. Investigations into virological and serological events that occur during the very early phase of HIV infection indicate that a high titre viraemia occurs generally during the second to third week after exposure (Figure I). This allows generalised seeding of the virus in susceptible tissue throughout the body. HIV genomic RNA is present before the patient has developed any detectable antiHIV antibodies $(1,2)$. Available evidence suggests that many new infections are caused by individuals unaware of their HIV infection (6). Rapid HIV test recently approved by FDA can help reduce unrecognized infections by improving access to test in a clinical setting. Rapid testing can play an important role in HIV prevention and expand access to testing in clinical settings. It can help overcome some of the barriers to early diagnosis and improve linkage to care of infected persons (3). In order to give quick results in case of worker result is given by a commercial HIV assay. The key-word is No waiting, this reduces anxiety and offers an accurate assessment in cases of occupational exposure (such as accidental needle prick) $(4,5)$. exposure during night shift or weekend work, a first rapid

Matrix HIV 1/2 Abbott ${ }^{\circledR}$ USA and ICD-HIV-p24Ag7 Innogenetics ${ }^{\circledR}$ USA for confirmation. Six of these 48 samples were selected because they also proved positive for Cytomegalovirus, Rubella Virus, Epstein Barr Virus, Herpes Simplex Virus 1 antibodies or elevated value of Total Bilirubin $(36 \mathrm{mg} / \mathrm{dL})$ to evaluate the analytical and diagnostic specificity of the test.

All samples were then investigated by the new POCT test $\mathrm{HIV} 1 / 2 \mathrm{Ag} / \mathrm{Ab}$ Combo Inverness Medical $^{\circledR} \mathrm{UK}$ : an immunochromatographic assay which yields results in less then 30 minutes.

\section{HIV Combo Ag/Ab Abbott Diagnostic ${ }^{\circledast}$ and Western Blot MATRIX HIV1/2 ABBOTT ${ }^{\circledR}$, USA}

This chemiluminescent microparticle immunoassay (CMIA) for the simultaneous detection of HIV p24 and HIV 1-2 antibodies in serum or plasma. It is a two step immunoassay to determine the presence of HIV p24 antigen and antibodies to HIV 1 (group $\mathrm{M}$ e $\mathrm{O}$ ) and HIV 2. The result is negative if reactivity is $<1.00$ Sample RLU/Cutoff RLU (S/CO); and is positive if the reactivity is $>=1.00 \mathrm{~S} / \mathrm{CO}$.

Western blot assay has been developed for confirmation and discrimination of both HIV-1 and HIV-2 antibodies.

The HIV-1/2 Western blot strips contain HIV-1 viral lysate and two recombinant envelope antigens, one for HIV-1 and one for HIV-2.

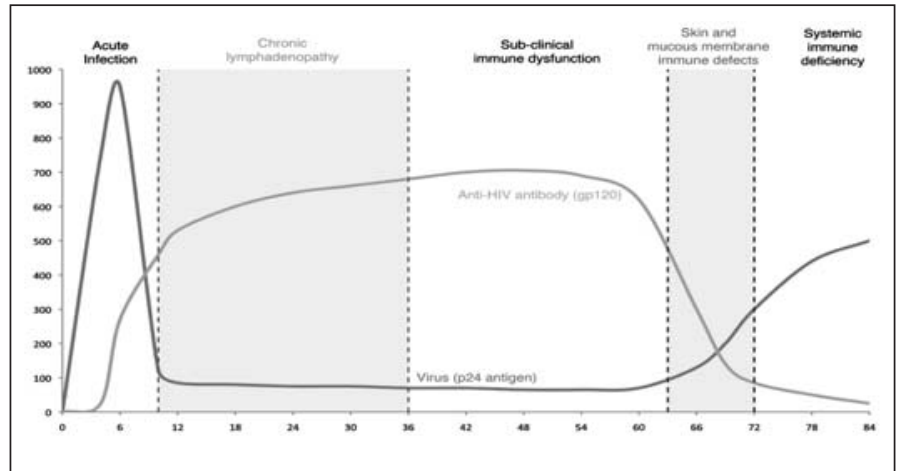

Figura I. Course of Ag and Abs in HIV infection.

\section{METHODS}

Forty-eight selected serum samples were previously analyzed, confirmed and classified for HIV positivity/negativity by conventional laboratory procedures: HIV Combo Ag/Ab Abbott Diagnostic ${ }^{\circledR}$ USA for screening and Western Blot
The three-hour assay uses an alkaline phosphatase conjugate and BCIP/NBT substrate. Use of this HIV-1/2 Western blot should significantly simplify confirmation of samples that are positive for HIV1/HIV-2 EIAs.

\section{HIV-1/2 Ag/Ab Combo Inverness Medical ${ }^{\circledR}$}

This is a qualitative immunochromatographic test for the simultaneous detection of HIV p24 antigen $(\mathrm{Ag})$ and antibodies $(\mathrm{Ab})$ to HIV-1 and HIV-2 in human serum, plasma, or wool blood.

The sample is added to the sample pad $(50 \mu 1$ and wait 30 minutes).

The specimen is mixed with a biotinylated anti-p24 antibody and selenium colloid-antigen conjugate.

This mixture continues to migrate through the solid phase to the immobilized avidin, recombinant antigens and synthetic peptides at the patient's window sites.

To ensure assay validity, a procedural control is incorporated into the device and is labelled control. If it is not red the test is invalid.

\section{Corresponding author: Marta Monari}

Laboratorio Analisi Cliniche, IRCCS Istituto Clinico Humanitas,

Via Manzoni 56, 20089 Rozzano, Milano - Tel.: 0282244763 - Fax: 0282244790

E-mail:marta_noemi.monari@humanitas.it 
Three different positive results can be obteined:

- Red bars appear both in the control window, in Ag window and in $\mathrm{Ab}$ window. In this case the double presence of $\mathrm{Ab}$ and Ag suggests an early stage of infection.

- If only one red bar shows in the Ag window, this suggests early infection.

- If only one red bar shows in the Ab window, this suggests past infection.

\section{RESULTS}

Forty-eight selected samples have registered these characteristics: 38 proved negative; 2 positive at the first step, but this result was not confirmed in WB, and 8 positive. We analyzed all them with the new POCT HIV1-2 Ag/Ab (Table 1). Analytical performance (sensitivity and specificity) and diagnostic accuracy of $100 \%$ were recordered.

It is noteworthy that the two serum resulting Ag-p24 positive by POCT were also confirmed by ELISA assay (ICD-HIVp24Ag7 Innogenetics ${ }^{\circledR}$, USA) (Table 1).

The evaluation of possible interferences due to EBV, CMV, Toxo, HSV, Rubeo Antibodies or elevated level of total bilirubin does not give false results.

Table I. Results of HIV detection tests.

\begin{tabular}{lccc}
\hline & $\begin{array}{c}\text { HIVI-2 Ag/Ab } \\
\text { COMBO }\end{array}$ & $\begin{array}{c}\text { Western Blot } \\
\text { or Ag-p24 }\end{array}$ & $\begin{array}{c}\text { HIVI-2 Ag/Ab } \\
\text { Rapid test }\end{array}$ \\
\hline POSITIVE & 10 & 8 & $2 \mathrm{Ag}+6 \mathrm{Ab}$ \\
\hline NEGATIVE & 38 & 40 & 40 \\
\hline
\end{tabular}

\section{CONCLUSION}

All critical healthcare settings should have access to an urgent HIV screening test which ideally gives results within four hours. Routine opt-out test results should be expected to be available within 72 hours. POCT gives a diagnostic evaluation on a possible source of infection in less than 30 minutes.

The complete concordance between rapid and conventional tests that detect the antigen and antibody of HIV, makes it possible to use POCT with great safety.

Enabling to reduce the inappropriate chemoprophylaxis due to long time of conventional laboratory investigations.

The current rapid point-of-care tests appear to be extremely valid for individual and selected people screening.

\section{REFERENCES}

1. Busch MP, Satten GA. Time course of viremia and antibody seroconversion following human immunodeficiency virus exposure. Am J Med 1997 ; 102 (5B): 117-24

2. Busch MP, Lee LL, Satten GA, et al. Time course of detection of viral and serologic markers preceding human immunodeficiency virus type 1 seroconversion: implications for screening of blood and tissue donors. Transfusion, 1995; 35 (2): 91-7.

3. Greenwald JL, Burstein GR, Pincus J, Branson B. A rapid review of rapid HIV antibody test. Inf Des Rep, 2006; 8: 125-34.

4. Lee-Lewadrowski E, Lewadrowski K. Perspectives on cost and outcomes for point-of-care testing. Clin Lab Med, 2009; 29 (3): 479-9.

5. Rapid HIV test kits. Health Devices, 2006; 35 (5): 157-77.

6. Samet JH, Fredeberg KA, Savetsky JB, et al. Understending delay to medical care for HIV infection: the long-term non presenter. AIDS, 2001; 15: 77-85.

7. www.iss.it - AIDS/HIV in Italia: rimane stabile il numero dei nuovi casi e aumenta la sopravvivenza dei malati 2008. 